\title{
The fate of magnetic fields in colliding galaxies
}

\author{
Hanna Kotarba ${ }^{1}$, Harald Lesch ${ }^{1}$, Klaus Dolag ${ }^{2}$ and Thorsten Naab ${ }^{2}$ \\ ${ }^{1}$ University Observatory Munich, Ludwig Maximilians University, \\ Scheinerstrasse 1, D-81679 Munich, Germany \\ email: kotarba@usm.lmu.de \\ ${ }^{2}$ Max Planck Institute for Astrophysics, \\ Karl-Schwarzschild-Str. 1, D-85741 Garching, Germany
}

\begin{abstract}
The evolution and amplification of large-scale magnetic fields in galaxies is investigated by means of high resolution simulations of interacting galaxies. The goal of our project is to consider in detail the role of gravitational interaction of galaxies for the fate of magnetic fields. Since the tidal interaction up to galaxy merging is a basic ingredient of cold-dark matter (CDM) structure formation models we think that our simulations will give important clues for the interplay of galactic dynamics and magnetic fields.
\end{abstract}

Keywords. galaxies: ISM, magnetic fields, interactions, methods: numerical, N-body simulations

\section{Introduction}

Radio observations reveal beyond any doubt that galaxies are permeated by ordered large-scale and irregular small scale magnetic fields, with spatial scales ranging from sizes of stars up to the spiral arm lengths, disk thickness and halo radii (e.g. Beck \& Hoernes, 1996, Chyży et al., 2007, Beck, 2009, Vollmer et al., 2010). Typical field strengths are of order of several $\mu \mathrm{G}$, ranging from a few $\mu \mathrm{G}$ in dwarfs (e.g. Chyży et al., 2003) up to $30 \mu \mathrm{G}$ in the star-forming regions of grand-design spiral galaxies (Fletcher et al., 2004). Magnetic fields have also been observed at high red-shifts like e.g. in damped Ly- $\alpha$ systems observed at red-shifts of $z \approx 0.4-2$. These systems, which have often been interpreted as large progenitors of present-day spirals (see Wolfe et al., 2005 for a review), seem to host similar $\mu \mathrm{G}$ magnetic fields (e.g. Bernet et al., 2008), or even magnetic fields of several tens of $\mu \mathrm{G}$ (Wolfe et al., 2008). The conversion of kinetic and turbulent energy into magnetic energy is a well investigated process in plasma astrophysics. The general term for this kind of energy transfer is "dynamo". It is commonly believed that dynamo processes like the turbulent dynamo and the $\alpha \Omega$-dynamo play a significant role in the evolution of magnetic fields (e.g. Kulsrud, 1999, Arshakian et al., 2009, and references therein). Those dynamo processes, however, rely on the presence of a "seed field" already existing at the onset of dynamo action. Those seed fields may be primordial, or a consequence of battery mechanisms in proto-galaxies or in the first stars. Primordial fields or battery processes could account for seed fields of $10^{-21}-10^{-18} \mathrm{G}$ (see e.g. Rees, 1987 and references therein), which would have to be amplified by more than ten orders of magnitude to reach the observed present-day values in galaxies. Therefore, other scenarios have been proposed. Within the most promising scenario, seed magnetic fields are built up during the lifetime of stars and expelled via relativistic winds during supernovae (SN) explosions (Rees, 1987). Those Crab-type SN remnants host magnetic fields of $\approx 10^{-4} \mathrm{G}$, pervading a volume of several cubic parsecs. Given that we would expect a huge number 
of stars forming in a protogalaxy, the mean field strength that could permeate the galaxy would be by orders of magnitude higher than that generated by the processes mentioned before. The galactic dynamo process could then reorder and further amplify this stellar seed field, while new born stars continue to seed magnetic fields into the galaxy. The seeding, reordering and amplification of those stellar seed fields has been recently shown by Hanasz (2009).

Since the evolution of large-scale magnetic fields is a part of galaxy evolution in general, the observations of remarkably strong magnetic fields even in very young galaxies raise the question about the origin and the evolution of the magnetic fields in our universe, especially in the context of hierarchical structure formation models. In these scenarios all present-day galaxies have undergone several major and minor mergers at early epochs and thereafter continuous accretion of gas and smaller galactic subunits (White \& Rees, 1978, White \& Frenk 1991, see Benson (2010) for a recent review). In other words, gravitational interactions are a key ingredient for the first phases of galaxy evolution. We can crudely estimate the amount of free energy during an interaction of two galactic subunits to be proportional to their relative velocity squared, i.e. $E_{\text {free }} \sim v_{\text {rel }}^{2}$. Obviously, some of this energy released during the interaction is converted into thermal energy of hot gas. High energy particles also carry away some of the energy. However, it is reasonable to assume that at least some of this energy is converted into magnetic field energy during the compression of gas and the formation of tidal structures. As the amount of $E_{\text {free }}$ can be very large during a major merger, the amount of energy converted into magnetic energy can be significant. Moreover, burst of star formation driven by the interaction (see e.g. Naab et al., 2006, Hopkins et al., 2008 and references therein) could continously seed new magnetic field, thus intensifying the amplification of magnetic fields even more.

Given the importance of the formation process for the present-day appearance of galaxies, there is a growing interest in the evolution of magnetic fields in the early universe (e.g. de Souza \& Opher 2010) and during galaxy formation (e.g. Dubois \& Teyssier, 2010, Wang \& Abel, 2009, Schleicher et al., 2010)

The dramatic impact of mergers on the gas flows will directly affect the dynamics of the magnetic fields of the systems. Since gas and magnetic field are tightly coupled, the magnetic field traces the gas motion and will be amplified by shocks and radial gas inflow. It is the aim of our work to shed light on the complicated nature of the evolution of magnetic fields in interacting systems. Understanding those systems is the first step towards a more complete understanding of the evolution of magnetic fields in the early universe.

\section{Simulations}

The simulations presented here were performed with the N-body/SPH-code GADGET (Springel, 2005). The dynamics of the Lagrangian fluid elements are followed using a SPH formulation including the evolution of magnetic fields which was implemented and tested by Dolag \& Stasyszyn 2009. See Kotarba et al. (2009) and Kotarba et al. (2010) for more details.

The Antennae galaxies.

A perfect example of the strong coupling of gas and magnetic fields is the interacting system NGC4038/4039 (the "Antennae galaxies"). We have simulated this system including magnetic fields for the first time (Kotarba et al., 2010a). The simulations demonstrated that even an initial magnetic field as small as $10^{-9} \mathrm{G}$ is efficiently amplified during the interaction of two equal-mass spiral galaxies. The strength of the magnetic 


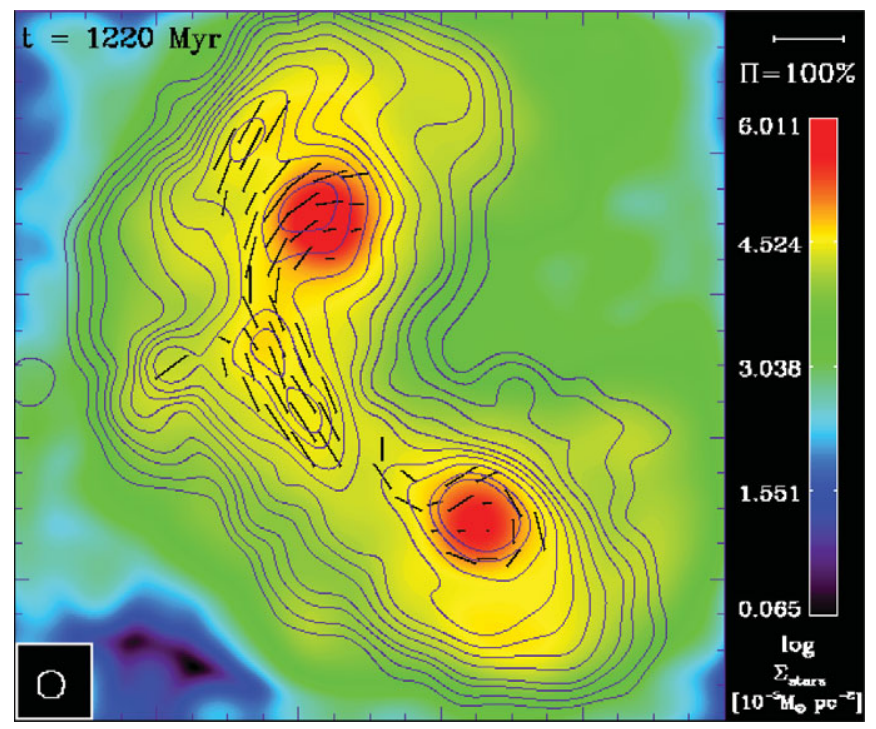

Figure 1. Artificial radio map of the simulated Antennae galaxies.

field thereby saturates at a value of $10 \mu \mathrm{G}$, independent of the initial magnetic field strength. This value compares well with the observations.

Fig. 1 shows an artificial radio map calculated at time of best match between the simulated gas and stellar distributions and observations. The radio map is in convincing morphological agreement with synchrotron observations of the Antennae system. Thus, the underlying numerics of the applied $N$-body/SPH code GADGET showed to be capable of following the evolution of magnetic fields in a highly nonlinear environment.

Saturation phenomena like that seen in our simulations usually occur when some kind of equipartition is reached. In case of the magnetic field, one usually assumes equipartition between the magnetic field energy density, the energy density of cosmic rays (CRs) and the energy density of the ionized gas. As we did not include CRs in our simulations, we have considered the energy densities of the gas. We have found that the estimated turbulent energy density of the gas is of the same order of magnitude as the magnetic field energy density. In view of the turbulent dynamo models which assume turbulence to be the main source of magnetic field amplification, this result seems reasonable. However, as we do not resolve the turbulent spectra of the gas in our simulations, but, instead, estimate the turbulence to be proportional to the rms velocity squared inside the SPH smoothing kernels, more studies are needed to assess the reason for the saturation of the magnetic field.

Ménage à trois.

We have performed simulations of three colliding galaxies to proof the saturation and equipartition phenomena seen in Kotarba et al., 2010a on the basis of a more general setup (Kotarba et al., 2010b). In these simulations, the magnetic field saturates at a value of about $1 \mathrm{G}$ - which is again consistent with observations -, independent of the initial value. Again, we find equipartition between the magnetic and the turbulent energy density.

Furthermore, the inclusion of the IGM in the simulations of colliding galaxies allows also for studying the magnetization of the IGM itself. We find that the shocks driven into the IGM by the interaction are the stronger, the higher the initial magnetic field within the galaxies and the IGM, suggesting that the shocks are gaining higher Mach numbers 
due to magnetic pressure support. Moreover, we find that there exists also a saturation magnetic field within the IGM, which has a mean value of $10^{-8}$ G. Again, this value agrees with observations.

Movies of the evolution of the magnetic field, the gas density, the temperature and the rms velocity als well as artificial radio maps for the "ménage à trois" can be found at http://www.usm.uni-muenchen.de/people/kotarba/public.html.

\section{Conclusion}

More detailed studies of galaxy interactions are needed to gain stronger insight into the nature of magnetic field saturation in interacting systems. However, on the basis of the performed simulations, we are confident that we are close to solve this issue.

Summing up our simulations clearly show that galactic interactions are efficient drivers of magnetic field amplification. All of the achieved saturation values agree with observations, thus indicating that these values develop naturally in real galaxies. On the basis of this work, we conclude that any galactic encounter in the history of the universe should have contributed to the magnetization of the universe. Within the CDM hierarchical structure formation model all galaxies build up through several major and minor mergers and the accretion of gas and smaller galactic subunits. Thus, within these models, the magnetization of the universe should be a natural part of galaxy formation and evolution.

\section{References}

Arshakian, T. G., Beck, R., Krause, M., \& Sokoloff, D. 2009, A $\& A$, 494, 21-32

Beck, R. \& Hoernes, P. 1996, NATURE, 379, 47-49

Beck, R. 2009, In: Magnetic Fields in the Universe II: From Laboratory and Stars to the Primordial Universe, ed. A. Esquivel, J. Franco, G. Garcï-Segura, E. M. de Gouveia Dal Pino, A. Lazarian, S. Lizano, \& A. Raga, RevMexAA, 36, 1-8

Benson, A. J. 2010, Physics Reports, 495, 33-86

Bernet, M. L., Miniati, F., Lilly, S. J., Kronberg, P. P., \& Dessauges-Zavadsky, M. 2008, NATURE, 454, 302-304

Chyży, K. T., Knapik, J., Bomans, D. J., Klein, U., Beck, R., Soida, M., \& Urbanik, M. 2003, $A \mathscr{E} A, 405,513-524$

Chyży, K. T., Bomans, D. J., Krause, M., Beck, R., Soida, M., \& Urbanik, M. 2007, A\&A, 462, 933-941

Dolag, K. \& Stasyszyn, F. 2009, MNRAS, 398, 1678-1697

Dubois, Y. \& Teyssier, R. 2010, A\&A, 523, A72+

Fletcher, A., Beck, R., Berkhuijsen, E. M., Horellou, C., \& Shukurov, A. 2004, In: How Does the Galaxy Work? A Galactic Tertulia with Don Cox and Ron Reynolds., ed. E. J. Alfaro, E. P+xiz, J. Franco, ASSL (Dordrecht: Kluwer), 315, 299-+

Hanasz, M., Wóltański, D., \& Kowalik, K. 2009, ApJL, 706, 155-159

Hopkins, P. F., Hernquist, L., Cox, T. J., Dutta, S. N. \& Rothberg, B. 2008, ApJ, 679, 156-181

Kotarba, H., Lesch, H., Dolag, K., Naab, T., Johansson, P. H., \& Stasyszyn, F. A. 2009, MNRAS, 397, 733-747

Kotarba, H., Karl, S. J., Naab, T., Johansson, P. H., Dolag, K., Lesch, H., \& Stasyszyn, F. A. 2010a, ApJ, 716, 1438-1452

Kotarba, H., Lesch, H., Dolag, K., Naab, T., Johansson, P. H., Donnert, J., \& Stasyszyn, F. A. 2010b, ArXiv e-prints, arXiv1011.5735

Kulsrud, R. M. 1999, ARAA, 37, 37-64

Naab, T., Jesseit, R., \& Burkert, A. 2006, MNRAS, 372, 839-852

Rees, M. J. 1987, QJRAS, 28, 197-206

Schleicher, D. R. G., Banerjee, R., Sur, S., Arshakian, T. G., Klessen, R. S., Beck, R., \& Spaans, M. 2010, $A \mathscr{E} A, 522, \mathrm{~A} 115+$ 
de Souza, R. S. \& Opher, R. 2010, PhRvD, 81, 6, 7301

Springel, V. 2005, MNRAS, 364, 1105-1134

Vollmer, B., Soida, M., Chung, A., Beck, R., Urbanik, M., Chyży, K. T., Otmianowska-Mazur, K., \& van Gorkom, J. H. 2010, A\&3A, 512, 36+

Wang, P. \& Abel, T. 2009, ApJ, 696, 96-109

White, S. D. M. \& Frenk, C. S. 1991, ApJ, 379, 52-79

White, S. D. M. \& Rees, M. J. 1978, MNRAS, 183, 341-358

Wolfe, A. M., Gawiser, E., \& Prochaska, J. X. 2005, ARAA, 43, 861-918

Wolfe, A. M., Jorgenson, R. A., Robishaw, T., Heiles, C., \& Prochaska, J. X. 2008, NATURE, $455,638-640$ 